\title{
Solving the maximum edge weight clique problem via unconstrained quadratic programming
}

\author{
Bahram Alidaee ${ }^{a}$, Fred Glover ${ }^{b}$, Gary Kochenberger ${ }^{\mathrm{c}, *}$, Haibo Wang ${ }^{\mathrm{d}}$ \\ a School of Business, University of Mississippi, United States \\ ${ }^{\mathrm{b}}$ University of Colorado at Boulder, United States \\ ${ }^{\mathrm{c}}$ School of Business, University of Colorado at Denver, CO, United States \\ d College of Business Administration, Texas A\&M International University, United States
}

Received 18 August 2004; accepted 8 June 2006

Available online 7 September 2006

\begin{abstract}
The unconstrained quadratic binary program (UQP) is proving to be a successful modeling and solution framework for a variety of combinatorial optimization problems. Experience reported in the literature with several problem classes has demonstrated that this approach works surprisingly well in terms of solution quality and computational times, often rivaling and sometimes surpassing more traditional methods. In this paper we report on the application of UQP to the maximum edge-weighted clique problem. Computational experience is reported illustrating the attractiveness of the approach. (c) 2006 Elsevier B.V. All rights reserved.
\end{abstract}

Keywords: Metaheuristics; Combinatorial optimization; Integer programming

\section{Introduction}

One of the tenets of combinatorial optimization is that given a choice, linear representations are preferred to nonlinear model forms. This preference for linearity is well entrenched in the conventional wisdom of both the theory and practice of combinatorial optimization and in fact has served the optimization community well over the years. Modern solution approaches like branch and cut and other methods designed to take full advantage of linearity

\footnotetext{
${ }^{*}$ Corresponding author.

E-mail addresses: Balidaee@bus.olemiss.edu (B. Alidaee), Fred.Glover@Colorado.edu (F. Glover), Gary.Kochenberger@ Cudenver.edu (G. Kochenberger), hwang@tamiu.edu (H. Wang).
}

have proven to be successful in practice, further underscoring the wisdom of the "linear choice". Linear representations also facilitate theoretical work aimed at understanding polyhedral (and other) properties of certain problems.

Opting for linearity may not, however, be the best choice from a computational point of view in all cases. This is particularly true for certain combinatorial problems that appear in their most natural form as nonlinear models but are "linearized" to enable the use of well-known solution methods designed for linear models. Successes with recent advances in metaheuristics and other solution approaches applicable to nonlinear models are calling the universality of the tenet of linearity into question. In this paper, we illustrate this notion by examining 
the maximum edge-weighted clique problem, a wellknown NP-hard problem. As detailed in several recent articles, this problem is typically solved in its linear form even though its most natural formulation is nonlinear.

In the sections below we give the natural, nonlinear formulation for the maximum edge-weighted clique problem followed by the linear version commonly appearing in the literature. We then comment on the metaheuristic procedure we use to solve the problem followed by some computational experience comparing our approach with recently published results. We conclude with a brief summary and some final remarks.

\section{Problem definition}

The maximum edge-weighted clique problem (MEWCP) can be defined as follows: Given a complete graph $G=(V, E)$ with $n$ nodes and unrestricted edge weights $c_{i j}$, find a subclique of $G$ with $b$ or fewer nodes such that the sum of the weights in the subclique is maximized. Since a given edge weight is included in the sum only if the associated pair of nodes is in the subclique, a natural, nonlinear formulation of this problem is

$$
\begin{aligned}
\text { Quadratic model: } & \max \\
& \sum_{i=1}^{n-1} \sum_{j=i+1}^{n} c_{i j} x_{i} x_{j} \\
\text { s.t. } & \sum_{j=1}^{n} x_{j} \leqslant b, \\
& x_{i} \in\{0,1\},
\end{aligned}
$$

where $x_{j}$ equals 1 if node $j$ is in the subclique; else $x_{j}$ equals 0 . This formulation is equivalent to that considered by Mehrotra (1997). We note that this model is of the form of the unconstrained quadratic binary program (UQP)

$$
\begin{gathered}
\mathrm{UQP}: \quad \max x Q x \\
x \text { binary }
\end{gathered}
$$

with the addition of a single cardinality constraint. This observation motivates the use of efficient metaheuristic methods for solving UQP to be applied to the problem considered here. The attractiveness of this approach is illustrated in Section 4 of this paper.

While the above model appears to be a natural representation of the problem, several solution methods proposed in the literature for solving MEWCP are based not on the quadratic model but instead on an equivalent linearization of the form (see for instance (Macambira and de Souza, 2000)):

$$
\begin{aligned}
\text { Linear model: } \max & \sum_{i, j, i<j} c_{i j} y_{i j} \\
\text { s.t. } \quad & y_{i j} \leqslant x_{i} \quad \forall(i, j) \in E, i<j \\
& y_{i j} \leqslant x_{j} \quad \forall(i, j) \in E, i<j \\
& x_{i}+x_{j}-y_{i j} \leqslant 1 \quad \forall(i, j) \in E, i<j \\
& \sum_{j \in V-\{i\}} y_{i j}-(b-1) x_{i} \leqslant 0 \quad \forall i \in V \\
& y_{i j}, x_{i} \in\{0,1\},
\end{aligned}
$$

where $x_{j}$ is defined as in quadratic model above and $y_{i j}$ equals 1 if edge $(i, j)$ is in the subclique; else $y_{i j}$ equals zero.

Note that the quadratic model is strictly a nodebased model consisting of $n$ variables and a single constraint. The linear model, which is obtained from the quadratic model via standard procedures for linearizing a quadratic function in binary variables, is both node and edge-based, consisting of $n+n(n-1) / 2$ variables and $n+3 n(n-1) / 2$ constraints. Thus, the linear model, while amenable to solution strategies designed for linear models, is much larger than the quadratic model. The differences in size and structure between the two equivalent models make a significant difference in the ease with which they can be solved. We illustrate this in the sections below.

\section{Solving MEWCP}

While MEWCP is NP-hard, considerable progress has been made in recent years in designing and testing exact methods intended to solve the linear model. Three recent articles standout as representative of these advances. Macambira and de Souza (2000) report on a branch and cut algorithm based on extensions of earlier work by Boros and Hammer (1993) and the work by Mehrotra (1997). They report computational experience with a set of test problems that have become a standard test bed for other researchers in the area. Hunting et al. (2001) report on a Lagrangian Relaxation approach that combines standard Lagrangian methods with cutting planes yielding a new approach to MEWCP. Finally, Sorensen (2004) reports on a new branch and cut method based on new classes of facet defining inequalities for the associated b-clique polytope. The later two papers report 
computational experience with the same problems introduced by Macambira and de Souza.

\subsection{Tabu search heuristic for quadratic model}

We solve instances of MEWCP directly in the nonlinear form of the quadratic model by applying a tabu search (TS) heuristic designed for the general, unconstrained binary quadratic programs (UQP). This approach, as indicated below, implicitly enforces the cardinality constraint and thus can be directly applied to MEWCP. ${ }^{1}$ An overview of our method, which proved to be very successful on standard MEWCP test problems, is given below:

Our TS method for UQP is centered around the use of strategic oscillation, which constitutes one of the primary strategies of tabu search. The method alternates between constructive phases that progressively set variables to 1 (whose steps we call "add moves") and destructive phases that progressively set variables to 0 (whose steps we call "drops moves"). To control the underlying search process, we use a memory structure that is updated at critical events, identified by conditions that represent locally optimal solutions that restrict the number of variables currently set to 1 to satisfy the cardinality condition $\left(\sum_{j=1}^{n} x_{j} \leqslant b\right)$. Solutions corresponding to critical events are called critical solutions. Additional moves on either side of a critical event, which degrade the critical solution and which may violate the cardinality constraint, are executed as part of the strategic oscillation employed.

A parameter span is used to indicate the amplitude of oscillation about a critical event. We begin with span equal to 1 and gradually increase it to some limiting value. For each value of span, a series of alternating constructive and destructive phases is executed before progressing to the next value. At the limiting point, span is gradually decreased, allowing again for a series of alternating constructive and destructive phases. When span reaches a value of 1, a complete span cycle has been completed and the next cycle is launched. The search process is typically allowed to run for a pre-set number of span

\footnotetext{
${ }^{1}$ Note, however, that the cardinality constraint found in the quadratic model could be brought into the $Q$ matrix by replacing the constraint by a quadratic infeasibility penalty, yielding an equivalent unconstrained version of the quadratic model. In turn, this unconstrained version of the quadratic model could be solved by any solution method designed for the unconstrained binary quadratic program.
}

cycles and the best solution found during this search process is then reported.

Information stored at critical events is used to influence the search process by penalizing potentially attractive add moves (during a constructive phase) and inducing drop moves (during a destructive phase) associated with assignments of values to variables in recent critical solutions. Cumulative critical event information is used to introduce a subtle long term bias into the search process by means of additional penalties and inducements similar to those discussed above. Other standard elements of tabu search such as short and long term memory structures are also included. A detailed explanation of this method us given in Glover et al. (1998) and Glover et al. (1999).

This basic implementation has been employed to solve a wide variety of combinatorial problems, some appearing naturally in the form of UQP and many others re-cast into the unified framework of UQP via the use of quadratic infeasibility penalties (see, for instance, Kochenberger et al., 2004a,b). We note that other heuristic approaches have recently been reported in the literature for a restricted version of MEWCP where all edge weights are required to be positive. (See Macambira, 2003 and Macambira and de Meneses, 1998.) These methods are not applicable to the general case of MEWCP considered here nor have comparisons been given by their authors with the methods considered here for solving the linear version of MEWCP. For these reasons, they are not considered further in this paper.

\section{Computational experience}

Our basic tabu search heuristic was used to solve a set of standard test problems originally appearing the paper by Macambira and de Souza (2000). Specifically, we solved the 30 test problems having both positive and negative edge weights. The results of our algorithm (denoted by TS/UQP) and those of Macambira and de Souza (M\&d), Hunting, Faigle and Kern (HFK) and Sorensen are given in Table 1.

The first two columns of the table give the problem identification and the known optimal solution for each problem. The next four columns give the computational times required for the four methods being considered. The times (seconds) given for M\&d, HFK and Sorensen are taken directly from their respective papers. The times reported for our UQP approach were obtained by running our heu- 
Table 1

Test problems with unrestricted edge weights from Macambira and de Souza

\begin{tabular}{|c|c|c|c|c|c|}
\hline ID & Optimal $X_{0}$ & M\&d time (seconds) ${ }^{\mathrm{a}}$ & HFK time (seconds) ${ }^{b}$ & Sorensen time (seconds) ${ }^{\mathrm{c}}$ & TS/UQP time (seconds) \\
\hline Grafo 40.1 & 70,348 & 12,481 & 14,079 & 223 & $<1$ \\
\hline 40.2 & 45,404 & 2219 & 1857 & 68 & $<1$ \\
\hline 40.3 & 34,091 & 1298 & 1129 & 64 & $<1$ \\
\hline 40.4 & 27,758 & 4759 & 2548 & 84 & $<1$ \\
\hline 40.5 & 27,967 & 477 & 2785 & 44 & $<1$ \\
\hline Grafo 42.1 & 81,633 & 18,754 & 14,841 & 269 & $<1$ \\
\hline 42.2 & 46,828 & 5569 & 4159 & 193 & $<1$ \\
\hline 42.3 & 36,689 & 1119 & 2130 & 84 & $<1$ \\
\hline 42.4 & 35,987 & 66 & 534 & 58 & $<1$ \\
\hline 42.5 & 35,460 & 707 & 1800 & 59 & $<1$ \\
\hline Grafo 44.1 & 90,620 & 20,388 & 27,445 & 347 & $<1$ \\
\hline 44.2 & 56,960 & 4201 & 3329 & 195 & $<1$ \\
\hline 44.3 & 40,697 & 1277 & 2142 & 151 & $<1$ \\
\hline 44.4 & 32,601 & 14,388 & 4208 & 169 & $<1$ \\
\hline 44.5 & 29,407 & 2633 & 1502 & 129 & $<1$ \\
\hline Grafo 45.1 & 102,295 & 16,111 & NA & 252 & $<1$ \\
\hline 45.2 & 55,103 & 11,021 & NA & 353 & $<1$ \\
\hline 45.3 & 43,914 & 637 & NA & 84 & $<1$ \\
\hline 45.4 & 33,990 & 7549 & NA & 140 & $<1$ \\
\hline 45.5 & 30,974 & 9397 & NA & 237 & $<1$ \\
\hline Grafo 46.1 & 99,550 & 19,276 & 10,348 & 383 & $<1$ \\
\hline 46.2 & 58,361 & 5988 & 4579 & 358 & $<1$ \\
\hline 46.3 & 43,915 & 7323 & 5418 & 242 & $<1$ \\
\hline 46.4 & 32,698 & 20,632 & 10,185 & 344 & $<1$ \\
\hline 46.5 & 31,000 & 1693 & 2350 & 144 & $<1$ \\
\hline Grafo 48.1 & 113,478 & 63,603 & 55,917 & 800 & $<1$ \\
\hline 48.2 & 61,768 & 33,527 & 36,963 & 840 & $<1$ \\
\hline 48.3 & 45,941 & 6625 & 3277 & 290 & $<1$ \\
\hline 48.4 & 36,903 & 2781 & 2257 & 206 & $<1$ \\
\hline 48.5 & 31,351 & 24,048 & 4505 & 307 & $<1$ \\
\hline
\end{tabular}

\footnotetext{
a SUN SPARC 1000 (Specific processor type not specified by authors).

b HP9000/735(125 MHz).

c Win95 on $350 \mathrm{MHz}$ PC (Specific processor type not specified by authors).

${ }^{\mathrm{d}}$ Intel Pentium $41.96 \mathrm{GHz}$.
}

ristic on a $1.96 \mathrm{GHz} \mathrm{PC}$ for an arbitrary limit of 50 SPAN cycles. Note that all methods found optimal solutions for all problems. ${ }^{2}$

A comparison of the computational times for the various methods must be done with all the usual caveats because the authors have used different machines. Additional care must also be exercised because our TS/UQP approach is heuristic in nature while the first three approaches are exact methods. Sorensen, making adjustments for the relative speed of the computers used, concludes that the time performance of M\&d and HFK are roughly equivalent

\footnotetext{
${ }^{2}$ Hunting, Faigle and Kern did not report results for the 45 node problems.
}

and that his method (on a $350 \mathrm{MHz}$ PC) shows improvement over that of M\&d by a factor of 10. M\&d and Sorensen did not report their processor type, and thus it is impossible to make precise timing comparisons with their results. However, by using the standard SPEC benchmark (http:// www.specbench.org/osg/cpu20000) we can conclude that the computer we used to produce our results is approximately three times faster than the computer used by HFK.

Whatever reasonable adjustments that might be made regarding comparable speeds of various computers, it is clear from Table 1 that the computation times for our approach to these test problems is very attractive. Our TS/UQP approach easily identified the optimal solution for each of the 30 problems 
by a search process conducted over an arbitrary limit of 50 SPAN cycles. The test problems considered here, corresponding to graphs with fewer than 50 nodes, are considered to be very small by UQP standards and the 50 SPAN cycles were executed in less than 1 second for each problem.

It is interesting to note that most of the time consumed by the LP-based methods applied to the linear model was spent on solving the initial LP relaxations. In fact for the two branch and cut methods (M\&d and Sorensen), each of the 30 problems were optimally solved at the root node and no branching was required. Moreover, the Lagrangian/Cut method of HFK required very few branches to solve these problems. As noted in Section 1, the linear model has many variables and constraints compared to the quadratic model and even for small instances like those considered here, the linear model generates large LPs that are time consuming to solve.

The approach by Sorensen appears to give the best performance of the three exact methods considered here. It is expected, however, that this approach (and indeed all the methods applied to the linear model) would degrade sharply in performance due to LP (and other) difficulties for larger problem instances. In contrast to this, the quadratic model scales nicely enabling much larger instances to be efficiently solved. For example, we have solved instances of MEWCP for graphs with $n=2000$ nodes in just a few minutes with our UQP heuristic. Problems of this size yield instances of the linear model with roughly $6,000,000$ constraints, ruling out the use of this representation while posing no problem at all for the quadratic model and our heuristic approach.

\section{Summary and conclusions}

In this paper, we have focused on the attractiveness of solving MEWCP directly in its nonlinear form rather than the more common approach of constructing and solving the equivalent but larger linear model. Modern metaheuristic methods, like critical event tabu search, enable the nonlinear version of MEWCP to be quickly solved. Even for small graphs as found in the available test bed, the computational advantage of the nonlinear model over the linear model is apparent. For instances of MEWCP defined on larger graphs, the attractiveness of the nonlinear model is even more pronounced. In fact, the linear representation of MEWCP may simply be unsolvable for even modest sized graphs (few hundred nodes) while the nonlinear model, approached by metaheuristic methods, can readily be applied to problems with several thousand nodes.

Adopting the nonlinear representation enables the state of the art in solving MEWCP to leap from problems with less than 100 nodes (according to the current literature) to those with several thousand nodes. As noted in recent papers (see for instance (Alidaee et al., 2005, 2006; Kochenberger et al., 2005a, 2004a,b, 2005b), the model UQP has proven to function efficiently and effectively as a unified framework for modeling and solving a wide variety of combinatorial optimization problems. In the context of other problem classes, we have solved instances of UQP with more than 50,000 variables, which means we could conceivably solve the nonlinear version of MEWCP for graphs with more than 50,000 nodes. As part of our future work, we plan to report on larger instances of MEWCP as well as a more detailed comparison with other heuristic methods. Our main objective here was to present the UQP approach for modeling and solving MEWCP and to illustrate its attractiveness by solving publicly available test problems.

\section{References}

Alidaee, B., Glover, F., Kochenberger, G., Rego, C., 2005. A new modeling and solution approach for the number partitioning problem. Journal of Applied Mathematics and Decision Sciences 9, 113-121.

Alidaee, B., Kochenberger, G., Lewis, K., Lewis, M., Wang, H., 2006. Modeling and solving set packing problems via unconstrained quadratic programming, Working Paper, University of Colorado at Denver.

Boros, E., Hammer, P., 1993. Cut-polytopes, Boolean quadratic polytopes and nonnegative quadratic pseudo-Boolean functions. Mathematics of OR 18, 245-253.

Glover, F., Kochenberger, G.A., Alidaee, B., 1998. Adaptive memory tabu search for binary quadratic programs. Management Science 44, 336.

Glover, F., Kochenberger, G., Alidaee, B., Amini, M., 1999. Tabu search with critical event memory: An enhanced application for binary quadratic programs. In: Voss, S., Martello, S., Osman, I., Roucairol, C. (Eds.), Meta-Heuristics, Advances and Trends in Local Search Paradigms for Optimization. Kluwer, pp. 93-109.

Hunting, M., Faigle, U., Kern, W., 2001. A Lagrangian relaxation approach to the edge-weighted clique problem. European Journal of Operational Research 131, 119-131.

Kochenberger, G., Glover, F., Alidaee, B., Rego, C., 2004a. Solving combinatorial optimization problems via reformulation and adaptive memory metaheuristics. In: Goldberg, A.M.a.D. (Ed.), Revolutionary Visions in Evolutionary Computation. Kluwer Publisher. 
Kochenberger, G., Glover, F., Alidaee, B., Rego, C., 2004b. A unified modeling and solution framework for combinatorial optimization problems. Operations Research Spectrum 26, 237-250.

Kochenberger, G., Glover, F., Alidaee, B., Lewis, K., 2005a. Using the unconstrained quadratic program to model and solve max 2-Sat problems. International Journal of Operational Research 1, 89-100.

Kochenberger, G., Glover, F., Alidaee, B., Rego, C., 2005b. An unconstrained quadratic binary approach to the vertex coloring problem. Annals of OR 139, 229-241.

Macambira, E.M., 2003. An application of tabu search heuristic for the maximum edge-weighted subgraph problem. Annals of OR 117, 175-190.
Macambira, E.M., de Meneses, C.N., 1998. A GRASP for the maximum edge-weighted subgraph problem. IX Confresso Latino-Iberoamericano de Investigacion Operativa; University of Buenos Aires.

Macambira, E.M., de Souza, C.C., 2000. The edge-weighted clique problem: valid inequalities, facets and polyhedral computations. European Journal of Operational Research 123, 346-371.

Mehrotra, A., 1997. Cardinality constrained Boolean quadratic polytope. Discrete Applied Mathematics 79, 137-154.

Sorensen, M., 2004. New facets and a branch-and-cut algorithm for the weighted clique problem. European Journal of Operational Research 154, 57-70. 